\title{
Las Relaciones Internacionales en México
}

\author{
Ana Covarrubias*
}

La Licenciatura en Relaciones Internacionales (RR.II.) es, hoy por hoy, uno de los programas universitarios más populares en México: se ofrece en un sinnúmero de universidades y hay variaciones que también atraen a muchos jóvenes (negocios internacionales, comercio internacional, relaciones económicas internacionales, etc.). De igual manera, los egresados de esta carrera se emplean en ámbitos muy distintos, desde el Servicio Exterior Mexicano, hasta empresas, organizaciones de la sociedad civil (osc), instituciones internacionales, el servicio público, y otros más. Como es de esperarse, el desarrollo de la Licenciatura en México se ha visto influido por condiciones internas e internacionales, y por las pautas que ha marcado la academia anglosajona principalmente.

Este ensayo describirá brevemente el origen y desarrollo de la enseñanza de las Relaciones Internacionales en México y presentará algunas reflexiones, con el perdón del lector, de naturaleza personal derivadas de $\mathrm{mi}$ experiencia como estudiante y profesora-investigadora en uno de los centros de investigación y docencia más reconocidos en el país: el Centro de Estudios Internacionales (CEI) de El Colegio de México. Estas reflexiones, sin embargo, no son ajenas al estado de la disciplina en el mundo; como se verá, reflejan mucho los cuestionamientos y cambios en la academia en general.

$$
* * *
$$

La enseñanza y la investigación de las Relaciones Internacionales en México ha sido poco estudiada. Los dos libros más recientes al respecto son el de Luis Ochoa Bilbao, La Carrera de Relaciones

Licenciada en Relaciones Internacionales por el Colegio de México. Posee una Maestría y un Doctorado en Relaciones Internacionales por la Universidad de Oxford (Gran Bretaña). En la actualidad, se desempeña como académica e investigadora del Centro de Estudios Internacionales (CEI) del Colegio de México. 
Internacionales en México. Orígenes y situación actual; ${ }^{1}$ el de Luis Ochoa Bilbao, Jorge A. Schiavon, Marta Tawil Kuri y Rafael Velázquez Flores (eds.), La disciplina de las Relaciones Internacionales en México: Enseñanza, enfoques $y$ programas docentes ${ }^{2}$, por supuesto, la parte correspondiente del de Arlene Tickner, Los estudios internacionales en América Latina. ¿Subordinación intelectual o pensamiento emancipatorio?, ${ }^{3}$ que serán la fuente principal para este ensayo.

El origen de la licenciatura fueron los estudios diplomáticos: en 1940, la Universidad Femenina (que fue absorbida por la Universidad del Valle de México en los años noventa), ofreció una carrera técnica de Estudios Diplomáticos pero, a decir de Luis Ochoa, fue realmente el Mexico City College (fundado en 1940) y después la Universidad Nacional Autónoma de México (UNAM, fundada en 1910), en donde realmente nació la Licenciatura en Relaciones Internacionales en la década de los cincuenta - aunque también como estudios diplomáticosEn efecto, el Mexico City College fue

1 México, El Colegio de México y Benemérita Universidad Autónoma de Puebla, 2011 (Jornadas 157).

2 México, Benemérita Universidad Autónoma de Puebla, Asociación Mexicana de Estudios Internacionales, Facultad de Economía y Relaciones Internacionales y Universidad Autónoma del Estado de Baja California, 2013.

3 Bogotá, Universidad de los Andes, ceso, Departamento de Ciencia Política, Alfaomega Colombiana, 2002. la primera institución que ofreció la carrera después de haber creado el Departamento de Relaciones Internacionales y Diplomacia. Se trata, sin duda, de un nacimiento muy singular pues el Mexico City College fue creado para formar diplomáticos estadounidenses (con una orientación hacia América Latina); es decir, se trataba de una institución privada fundada principalmente para atender a los estadounidenses que vivían en México (y a algunos mexicanos). No deja de ser irónico que la "disciplina americana", como la llamó Stanley Hoffmann, haya llegado a México de esta manera, y con el objetivo de preparar diplomáticos extranjeros. Según Ochoa, la disciplina adquiriría "personalidad propia” en el Mexico City College, y se consolidaría en los años sesenta.

Dos programas más surgirían en los años cincuenta y sesenta en instituciones públicas, con el objetivo primordial,también, de capacitar cuadros mexicanos para el servicio exterior: se trata de la unam y El Colegio de México (fundado en 1938 como la Casa de España en México). En el primer caso, la Escuela Nacional de Ciencias Políticas y Sociales (ENCPS) inició actividades en 1951 y ofreció la carrera de Ciencias Diplomáticas.

Tal como sucedería posteriormente con El Colegio de México, las licenciaturas respondían a las necesidades de un Estado fuerte que contaba con cierta actividad internacional, sobre todo en la unesco y otros órganos del sistema de Naciones Unidas, aunque 
su política exterior se caracterizara por ser más bien pasiva y muy legalista (lo cual, como lo demuestra Ochoa, se refleja claramente en el tipo de publicaciones sobre Relaciones Internacionales de la época, algunas de ellas producidas por diplomáticos). Así pues, El Colegio de México ofrece por primera vez la licenciatura, esta sí en Relaciones Internacionales como tal, en 1960, cuando se crea el Centro de Estudios Internacionales (CEI) un poco después de la publicación de la revista Foro Internacional. El interés de su fundador, Daniel Cosío Villegas, era formar funcionarios especializados para un país que enfrentaba a un escenario de Guerra Fría, pero también de creciente interdependencia. Para Cosío Villegas, era importante que México tuviera un papel en la esfera mundial y no quedara ajeno a la historia.

La licenciatura de la UNAM se caracterizaba por un enfoque histórico y jurídico, y el plan de estudios se inspiró en los modelos de The London School of Economics, el Institute d'Etudes Politiques de la Universidad de París y de la École de Sciences Politiques e Sociales de la Universidad de Lovaina. En 1970 se creó el Centro de Relaciones Internacionales (CRI), y se inició la publicación del Boletín de Relaciones Internacionales que en 1973 se convirtió en la revista Relaciones Internacionales.

El programa de El Colegio de México-que se elaboró con asesoramiento de Kenneth W. Thompson, discípulo de Hans Morgenthau - fue interdisciplinario y se distinguió por un alto contenido de lo que hoy definiríamos como estudios regionales, pero no incluyó materias sobre México, excepto por una llamada "México y América Latina en el mundo internacional de hoy". Con el tiempo, el contenido de materias sobre México creció de manera significativa. Gracias a la actividad docente, la labor de investigación y la publicación de Foro Internacional, el CEI — en palabras de Ochoa- "se convirtió en un referente no solo para que el Estado se nutriera de cuadros profesionales para el servicio exterior, sino para la consolidación de las Relaciones Internacionales en México..." ${ }^{4}$

Por varios años, el Mexico City College, la unam y El Colegio de México fueron las instituciones que ofrecieron programas en Relaciones Internacionales y cumplieron con su función de capacitar profesionistas para el servicio exterior - que no era muy grande, dados los intereses limitados de México en el mundo-. Además, en 1974 se creó el Instituto Matías Romero, de la Secretaría de Relaciones Exteriores (SRE) para ofrecer cursos de preparación para el ingreso al servicio exterior. La apertura económica del país que inició en los años ochenta, después de la crisis de la deuda, cambiaría la perspectiva del estudio de las Relaciones Internacionales y contribuiría, junto con el fin de la Guerra Fría, a que la licenciatura se convirtiera en una de alta demanda. Así pues,

4

La carrera de Relaciones Internacionales en México, op.cit., p. 72. 
la apertura económica de México y la globalización modificaron la naturaleza de la licenciatura, que incluyó un contenido económico y financiero importante. Como resultado, empresas y organizaciones privadas, muchas de ellas del sector financiero, y un Estado con requerimientos distintos, demandaban especialistas en Relaciones Internacionales, lo cual diversificó la oferta de empleo para los egresados. Los programas de estudio ya establecidos se actualizaron y los nuevos se diseñaron para el mundo globalizado. La licenciatura dejó de estar dirigida principalmente a la formación de diplomáticos y funcionarios del Estado mexicano.

$* * *$

La Licenciatura en Relaciones Internacionales se volvió muy popular en México hacia finales de los años ochenta y durante la década de 1990. Las razones que explican este boom son simples, y ya se han mencionado: la apertura económica de México y el fin de la Guerra Fría. La apertura económica de México, que inicia con la adhesión del país al GATT en 1986, implicó cambios muy significativos en todos los ámbitos de la vida del país; lo que aquí importa es el hecho de que México estrechaba sus vínculos con una diversidad de actores internacionales en asuntos muy variados. De alguna manera, el mundo entraba al país y México se reposicionaba en el sistema internacional. En política interna, esto también produjo modificaciones notorias en términos del tipo de actores que se volvían indispensables para las relaciones internacionales del país. Esta situación fue evidente durante la negociación del Tratado de Libre Comercio de América del Norte (TLCAN), cuando la Secretaría de Comercio y Fomento Industrial (SECOFI), el Banco de México y la Secretaría de Hacienda y Crédito Público (SHCP), tomaron un papel protagónico en la política exterior de México, en detrimento del de la SRE. En segundo lugar, las consecuencias del fin de la Guerra Fría y el proceso de globalización en el sistema internacional y las políticas exteriores de los países son bien conocidas. Así pues, la casi coincidencia entre la apertura económica de México, el fin de la Guerra Fría - y posteriormente la apertura política - replantearon el estudio y la prominencia de las relaciones internacionales de México.Las conexiones del país con el mundo se multiplicaron en número y naturaleza, y requerían de profesionistas y diplomáticos para atender un sinfín de asuntos.

Entre los años ochenta y los noventa muchas universidades, públicas y privadas, en la Ciudad de México y otros estados de la República, abrieron programas de Relaciones Internacionales, entre ellas, la Benemérita Universidad Autónoma de Puebla (BUAP), el Centro de Investigación y Docencia Económicas (CIDE), El Colegio de San Luis, el Instituto Tecnológico Autónomo de México (IтAm), el Instituto 
Tecnológico y de Estudios Superiores de Monterrey (ITESM), la Universidad Anáhuac, la Universidad Autónoma del Estado de Baja California (UABC), la Universidad de Colima, la Universidad Iberoamericana (UIA), la Universidad del Mar, la Universidad Popular Autónoma del Estado de Puebla (UPAEP) y la Universidad de Quintana Roo (UQroo). El Mexico City College, que ya ofrecía la licenciatura, se dividió en la Universidad de las Américas y la Fundación Universidad de las Américas Puebla (UDLAP), y ambas instituciones continuaron ofreciendo la carrera. Por su naturaleza, los planes de estudio de prácticamente todas ellas son multidisciplinarios y dan un lugar especial a los estudios regionales, aunque cada una de ellas cuenta con particularidades y especializaciones. Menciono algunos ejemplos: la Universidad Iberoamericana busca formar profesionistas para "incidir en el diseño de políticas, estrategias e instrumentos prácticos que contribuyan a la solución de problemas sociales globales." Al tratarse de una universidad confesional (jesuita), el compromiso y la responsabilidad social son esenciales en la preparación de los estudiantes, por lo que procura que sus egresados sean capaces de diseñar y ejecutar políticas que promuevan la

5 Laura Zamudio González, "El estudio de las Relaciones Internacionales en la Universidad Iberoamericana", en Ochoa Bilbao, Schiavon, Tawil Kuri y Velázquez Flores (eds.), La disciplina de las Relaciones Internacionales en México, op, cit., pp.143-155. cooperación para atender problemas globales como la pobreza, la migración, los refugiados, la seguridad, el narcotráfico, el desarrollo ambiental sostenible, la justicia, los derechos humanos, etc. El plan de estudios incluye cuatro áreas de especialización terminal: política y derecho internacional, comercio internacional, medio ambiente y Relaciones Internacionales, y cultura y Relaciones Internacionales. ${ }^{6}$ El IтAM, aprovechando la fortaleza de la institución en disciplinas como las matemáticas y la economía, favorece la preparación cuantitativa de los estudiantes para que puedan entender los flujos financieros y comerciales globales, así como la lógica de actores económicos internacionales. En palabras de Natalia Saltalamacchia, "[E]l IтAM aspira a formar mexicanos universales, es decir, profesionistas con una visión y ambición global, pero capaces de pensar el mundo en y desde México"? Debe mencionarse que, al igual que la uDLAP, el estudio de los Estados Unidos ocupa un lugar destacado en el plan de estudios. Después de la firma del TLCAN, la udLAP —así como El Colegio de México- incluyó al programa materias sobre Canadá y, en los

6 Idem.

7 Natalia Saltalamacchia Ziccardi, "El estudio de las Relaciones Internacionales en el ITAM" y Marcela Álvarez Pérez, "La carrera de Relaciones Internacionales en la Universidad de las Américas Puebla”, en Ochoa Bilbao, Schiavon, Tawil Kuri y Velázquez Flores (eds.), La disciplina de las Relaciones Internacionales en México, op. cit., pp. 79-86 y 101-108, respectivamente. 
últimos años, también de Derecho y práctica consular y diplomática, con la intención de retomar los orígenes de la licenciatura que, como se ha mencionado, se orientaba a la educación de diplomáticos. ${ }^{8}$

El ITESM, por su parte, se propone formar líderes con "espíritu emprendedor, sentido humano y competitivos internacionalmente". Ofrece la licenciatura en alrededor de ocho campus y da a los estudiantes la oportunidad de tener experiencias prácticas a nivel internacional mediante intercambios, proyectos, cursos y congresos.

El Colegio de México, según Marta Tawil, mantiene una tradición metodológica europea que ofrece una alternativa a la dominante escuela estadounidense, aunque sin descuidar el estudio de Estados Unidos, sobre todo la relación bilateral y, en menor medida, Canadá. La licenciatura del CEI preserva un corte clásico y de carácter multidisciplinario, con un contenido humanista esencial en el que la historia, los estudios regionales y el derecho ocupan un lugar sobresaliente. ${ }^{10}$

8 Álvarez Pérez, loc. cit.

9 Luz Araceli González Uresti, "El Tecnológico de Monterrey: más de 20 años formando internacionalistas", en, Ochoa Bilbao, Schiavon, Tawil Kuri y Velázquez Flores (eds.), La disciplina de las Relaciones Internacionales en México, op. cit. pp. 87-100.

10 Marta Tawil Kuri, "El estudio actual de las Relaciones Internacionales en El Colegio de México”, en Ochoa Bilbao, Schiavon, Tawil Kuri y Velázquez Flores (eds.), La disciplina de las Relaciones Internacionales en México, op. cit., pp. 47-62.
El Colegio de México brinda la oportunidad de estudiar las Relaciones Internacionales como disciplina autónoma y como práctica política. ${ }^{11}$

La Escuela de Relaciones Internacionales de la Universidad Anáhuac empezó a funcionar en 1996. Cuenta también con materias regionales, una fuerte base teórica y áreas de pre-especialización: Economía y Globalización, Seguridad Internacional, Derechos Humanos y Medio Ambiente. En la UNAM, como en otras instituciones, la Licenciatura en Relaciones Internacionales es de alta demanda. Como se anotó más arriba, junto con el Mexico City College y El Colegio de México, el programa de la unam fue de los primeros en México y su objetivo fue formar diplomáticos. También como en los casos mencionados, a partir de los años sesenta se amplió su enfoque para preparar internacionalistas no solo para el sector público, sino también para el privado y las organizaciones civiles, tendencia que se ha fortalecido como resultado de los procesos de globalización, transnacionalización y mundialización. El mapa curricular actual cuenta con seis áreas disciplinarias: teórico-metodológica, de política, economía y derecho internacional, de política exterior de

11 Idem. 
México y de estudios regionales. ${ }^{12} \mathrm{El}$ CIDE, finalmente, difiere un poco de las universidades anteriores, pues ofrece la Licenciatura en Ciencia Política y Relaciones Internacionales y no únicamente en esta última disciplina. A decir de Jorge Schiavon, la carrera se propone educar profesionistas que puedan vincular variables internas e internacionales, de índole político, económico y social, para poder analizar y proponer soluciones a problemas urgentes que aquejan a las sociedades contemporáneas. Como en los demás casos, el plan de estudios es interdisciplinario, pero da un lugar importante a los modelos teóricos formales y a los instrumentos cuantitativos. ${ }^{13}$ Para terminar este breve recuento, vale la pena decir que algunas instituciones orientan la licenciatura según su ubicación geográfica y se especializan en temas como migración, comercio, problemas transfronterizos, y en regiones como Centroamérica y el Caribe, América

12 Javier Zarco Ledesma, "Un acercamiento a la enseñanza de las Relaciones Internacionales en la unAm", en Ochoa Bilbao, Schiavon, Tawil Kuri y Velázquez Flores (eds.), La disciplina de las Relaciones Internacionales en México, op. cit., pp. 169-185.

13 Jorge A. Schiavon, "La Licenciatura en Ciencia Política y Relaciones Internacionales (CP/RI) en el CIDE", en Ochoa Bilbao, Schiavon, Tawil Kuri y Velázquez Flores (eds.), La disciplina de las Relaciones Internacionales en México, op. cit., pp. 35-46. del Norte (principalmente Estados Unidos) o Asia Pacífico. ${ }^{14}$

El Cuadro I presenta el número de programas en Relaciones Internacionales (y sus variaciones) en México, según lo registra la Asociación Nacional de Universidades e Instituciones de Educación Superior (anuies). Como es evidente, lo que es realmente popular es la licenciatura, quizá por su carácter general. Hay pocas oportunidades para estudios de posgrado, sobre todo de doctorado. Esto, sin embargo, no quiere decir que la disciplina no se haya consolidado en México. Como se verá más adelante, hay centros de estudio de Relaciones Internacionales o temas afines y casas editoriales

14 Como es el caso de El Colegio de San Luis, la UABC, la Universidad de Colima, la Universidad del Mar y la de Quintana Roo. Véase, M. Cecilia Costero G. y Patricia Rubio Ortiz, "El estudio de las Relaciones Internacionales de El Colegio de San Luis: Una opción en la región centro-norte de México”; Ana Bárbara Ungaray Moctezuma, Saúl Oziel López Llanes y Rafael Velázquez Flores, "Enseñanza de las Relaciones Internacionales en la Universidad Autónoma de Baja California: Modelo por competencias"; María Elena Romero, "La enseñanza de las Relaciones Internacionales. El caso de la Facultad de Ciencias Políticas y Sociales de la Universidad de Colima"; Isaac Flores Delgado, "La Universidad del Mar: Un modelo de excelencia en la enseñanza e investigación de Relaciones Internacionales"; y Enrique Baltar Rodríguez, "La formación de internacionalistas en la Universidad de Quintana Roo: Experiencias, avances y desafíos", todos en Ochoa Bilbao, Schiavon, Tawil Kuri y Velázquez Flores (eds.), La disciplina de las Relaciones Internacionales en México, op. cit. 
que publican regularmente sobre te- disciplina siga siendo dominada por mas de asuntos internacionales. Pero los enfoques del "Norte". sí puede influir en el hecho de que la

\section{Cuadro I}

Programas de Educación Superior en Relaciones Internacionales

\begin{tabular}{|l|l|}
\hline \multicolumn{2}{|c|}{ NIVEL LICENCIATURA } \\
\hline Concepto & Total \\
\hline Licenciatura en Relaciones Internacionales & 90 \\
\hline Licenciaturas en Relaciones Internacionales y otra disciplina & 9 \\
\hline $\begin{array}{l}\text { Licenciatura en Relaciones Internacionales y Comercio } \\
\text { Exterior }\end{array}$ & 4 \\
\hline $\begin{array}{l}\text { Licenciaturas en Ciencias Políticas y Relaciones } \\
\text { Internacionales }\end{array}$ & 2 \\
\hline Licenciaturas en Negocios y Relaciones Internacionales & 2 \\
\hline $\begin{array}{l}\text { Licenciatura en Lenguas Extranjeras y Relaciones } \\
\text { Internacionales }\end{array}$ & 1 \\
\hline Matriculados en Licenciatura en Relaciones Internacionales & 23,746 \\
\hline \multicolumn{1}{|c|}{ NIVEL POSGRADo } & Total \\
\hline Concepto & 9 \\
\hline Posgrados en Relaciones Internacionales & 8 \\
\hline Maestrías & 1 \\
\hline Doctorados & \\
\hline
\end{tabular}

\begin{tabular}{|l|l|}
\hline Matriculados en Posgrados en Relaciones Internacionales & 166 \\
\hline
\end{tabular}

Fuente: http://www.anuies.mx/ informacion-y-servicios/informacionestadistica-de-educacion-superior/ anuario-estadistico-de-educacion-superior, consultada el 22 de septiembre de 2019.
La docencia y la investigación en Relaciones Internacionales en México se ha encontrado con los dilemas y cuestionamientos, muchos, a los que se ha enfrentado la disciplina en el ámbito internacional, especialmente 
en la academia anglosajona. Lo primero que debe decirse, en efecto, es que, a pesar de que la mayoría de los programas en México son de Relaciones Internacionales y no de Ciencia Política (lo cual supone que se le reconoce como disciplina independiente), el desarrollo de esta última ha permeado de manera profunda el de la primera. Identifico tres ejemplos: la creciente importancia de la metodología (y los tipos de métodos) y la teoría, y la necesidad de los estudios comparados. Hasta el día de hoy, sigue habiendo una discusión sobre el tipo de métodos que se deben usar para el análisis de las relaciones internacionales, discusión que quizá llegó a México tarde en los años setenta. En este sentido, hubo, y sigue habiendo, una división fuerte entre quienes arguyen que, por su naturaleza multidisciplinaria, en la que destacan la Historia y el Derecho, y humanista, el método debe ser principalmente cualitativo, y quienes consideran que son los métodos cuantitativos los que darán solidez científica a la disciplina. Es decir, se discute también la naturaleza científica - $\mathrm{O}$ no- de la disciplina y si es deseable aspirar a ello. En general, fueron los programas de licenciatura que iniciaron en los años noventa los que de entrada aceptaron la necesidad y la relevancia de los métodos cuantitativos, aunque los planes que ya existían se actualizaron para incluirlos, como fue el caso de El Colegio de México. Como se ha visto, el ITAm o el CIDE, por ejemplo, dan un lugar especial al conocimiento cuantitativo, lo que distingue y es la fortaleza de sus programas. Esta pluralidad de visiones, aunque parte de un debate muchas veces acalorado, es una enorme ventaja pues da a los estudiantes la posibilidad de escoger el que más se acerque a sus intereses.

En la actualidad, las publicaciones sobre Relaciones Internacionales y política exterior de México cubren una variedad de métodos, pero no deja de haber una cierta "competencia" sobre su tipo y el cuestionamiento constante sobre el dominio del método sobre el contenido.

El tema de la teoría es de sumo interés. Arlene Tickner vincula el desarrollo de la disciplina en América Latina con su posición de región periférica y arguye que el surgimiento y la consolidación de los estudios de Relaciones Internacionales fue justamente resultado de la búsqueda por disminuir los niveles de dependencia política, económica e intelectual de la región. Tickner sostiene que se necesitaba un pensamiento crítico ante los sucesos mundiales, alternativo a la teoría dominante, el Realismo, que ignoraba el contenido sustantivo y normativo de las relaciones internacionales y, en consecuencia, tenía poca relevancia 
para las relaciones de la región. ${ }^{15}$ Tickner analiza, así, el peso de la teoría de la Dependencia y, más importante aún, la literatura sobre autonomía producida en América Latina que, en sus palabras, "tendió un puente conceptual entre el análisis de la dependencia y las teorías tradicionales de las relaciones internacionales, sobre todo el realismo clásico y, más tarde, la interdependencia, al tiempo que trascendió las conclusiones pesimistas derivadas de la dependencia (y el realismo) en términos de la posibilidad de una acción autónoma por parte de los países débiles en el sistema internacional."16 $\mathrm{No}$ hay duda del lugar de la Dependencia en el estudio y la enseñanza de Relaciones Internacionales en México; en el caso de la autonomía, sin embargo, su uso como concepto analítico se ha dado principalmente en el estudio de la política exterior, como se verá más adelante. Lo cierto es que, en términos teóricos, la disciplina en México continúa siguiendo de cerca las propuestas de la academia anglosajona: Realismo (Neorrealismo), Liberalismo (Neoliberalismo) y Constructivismo y, cada vez más, la Escuela Inglesa. Es más difícil encontrar propuestas teóricas propias, tal como ha sucedido en América del

15 Arlente Tickner,Los estudios internacionales en América Latina. ¿Subordinación intelectual o pensamiento emancipatorio?, Bogotá, Universidad de los Andes, Ceso, Departamento de Ciencia Política, Alfaomega Colombiana, 2002, pp. 55-56.

16 Tickner, Los estudios internacionales en América Latina, op. cit., p. 56.
Sur, especialmente Argentina y Brasil (el Realismo periférico, por ejemplo,y la elaboración más sofisticada de la autonomía). Luciano Tomassini, citado por Tickner, fue muy claro en cuanto a la falta de producción teórica propia en México: "En el país se han realizado pocas reflexiones teóricas sobre relaciones internacionales, al tiempo que la participación en la "comunidad de relaciones internacionales" ha servido, en muchos casos, como trampolín para el análisis político, así como para participar en la vida pública.".

Otro tema que vale la pena mencionar es el lugar del estudio de Estados Unidos en la investigación y la docencia. Es cierto que el estudio de este país en cualquier programa de RR.II. en el mundo es indispensable, pero en el caso de México es prioritario - lo que es bueno y malo-. Como se ha mencionado, algunas universidades en la Ciudad de México y en otros estados de la República otorgan mucha importancia al conocimiento sobre Estados Unidos y la problemática que supone ser su vecino. Así pues, no es extraño encontrar planes de estudio con varias materias sobre Estados Unidos, y solo una sobre América Latina, por ejemplo, o ninguna sobre África. Lo que hay que destacar es el deseo explícito de algunas instituciones de dar una orientación especial a sus programas hacia Estados Unidos. Esto nos lleva a otro debate en la disciplina, sobre todo hacia el final de la Guerra Fría

17 Citado en Ibid., p. 84. 
y antes del 11 de septiembre: el valor de los estudios regionales que, como ya se dijo, es un componente esencial en muchas licenciaturas. Esta discusión se dio, en parte, como cuestionamiento al peso de la historia en los programas de RR.II., por un lado, y al fortalecimiento de los estudios comparados (Comparative Politics). ¿Qué es más útil y para qué: conocer un caso a profundidad o comparar dos, tres o múltiples países, problemáticas, etc.? Una vez más, hay voces que defienden el valor de conocer a fondo un país, una región, un tema, mientras que otras prefieren la comparación para poder generalizar y, en el mejor de los casos, teorizar. En el caso del CEI, se discutió la posibilidad de cambiar los estudios regionales por comparados, pero se decidió mantener el estudio de regiones pues no solo era una característica definitoria del programa (que ha sido muy exitoso), sino que sigue considerando que la historia y el conocimiento a profundidad de las regiones (en la medida de lo posible) es fundamental para entender las relaciones internacionales. El 11 de septiembre nos recordó, sin duda, el valor de los estudios regionales y no deja de sorprender que los programas descritos en el libro de Ochoa Bilbao, et. al, ya citado (15 entre instituciones públicas y privadas a lo largo del país), reproduzcan el modelo de plan de estudios con regiones. El cIDE es la institución que busca abiertamente fomentar la capacidad comparativa de los estudiantes, y no sorprende al tratarse de una licenciatura en Ciencia Política y Relaciones Internacionales.

Para terminar, cabe señalar que a pesar de la enseñanza de las teorías principales (Realismo, Liberalismo y Constructivismo) y de otras perspectivas como la Interdependencia o la Globalización, el estudio de las Relaciones Internacionales en México, según los planes de estudio revisados, tiende a ser Estado-céntrico, aunque cada vez más los estudios temáticos en los que sobresalen una variedad de actores y redes, adquieren importancia. Sin embargo, como se ha visto, la licenciatura se ofrece como Relaciones Internacionales y parece haber poco interés, por el momento, de convertirla en Estudios Globales.

$* * *$

Aunque la academia mexicana no se distingue por una producción teórica propia, lo cierto es que sí ha desarrollado un análisis conceptual de la política exterior de México, como era de esperarse, alegando en muchos casos su singularidad como resultado de la ubicación geográfica y, por lo tanto, geopolítica del país. Generalizando mucho, podríamos hablar de dos maneras mediante las cuales se ha estudiado tradicionalmente la política exterior de México: la que se enfoca en su naturaleza y, estrechamente vinculado con esta, la que subraya la posición/actuación del país en 
el sistema internacional. En relación con la primera, destaca la idea de "una política exterior de principios", que sirvió mucho a los gobiernos mexicanos, en especial como retórica, durante momentos difíciles de la Guerra Fría (aunque el presidente actual, Andrés Manuel López Obrador, ha reiterado su voluntad de implementar nuevamente una política de ese tipo). Con el tiempo, esta idea se convirtió en un debate entre principios e intereses, una falsa disyuntiva quizá que, sin embargo, permitió el uso de nuevos y variados enfoques para analizar la política exterior.

Aunado a la política de principios, durante muchos años el estudio de la política exterior de México destacó la idea de la autonomía e independencia como guías de conducción y objetivo de la política exterior (pero no como propuesta teórica tal como se hizo en América del Sur). Desde luego, el punto de referencia fundamental ha sido Estados Unidos: México tiene que distinguirse y actuar de forma independiente a como lo hace Estados Unidos. La firma del TLCAN, empero, comenzó a modificar un poco esta posición, en tanto cambió el papel de Estados Unidos, de amenaza a oportunidad. Esto no quiere decir que los gobiernos mexicanos no hayan procurado mantener una política exterior independiente pero, en ocasiones, se aceptó el hecho de que ambos países pudieran tener intereses similares. En otras palabras, la autonomía de la política exterior dejó de ser una camisa de fuerza.

La segunda manera de examinar la política exterior de México es la de "clasificar" al país, ya fuera como nación tercermundista, potencia media o regional, país emergente, actor con responsabilidad global, jugador internacional, etc. Aunque este tipo de análisis se inició durante la Guerra Fría, la voluntad explícita de México de perfilarse como actor regional y global se dio gracias a la globalización y a la apertura política en el país. Para los gobiernos mexicanos había que ser parte de una categoría de países que reflejara su voluntad $y$ capacidad de actuar y, sobre todo, influir, en el escenario internacional. Sin embargo, actualmente el deseo de la SRE de mantener lo que en política exterior mexicana se ha calificado tradicionalmente como una política "activa" se encuentra con la indiferencia presidencial hacia la política exterior en general, por lo que es difícil afirmar que México mantiene el interés de ser jugador global.

Para el estudio más sofisticado de la política exterior se ha recurrido a la subdisciplina de Análisis de Política Exterior pero, como en el caso de la teoría, frecuentemente es difícil su aplicación directa al tratarse de modelos de países del Norte. Los procesos de toma de decisión à la Allison, por ejemplo, son sumamente difíciles de rastrear y demostrar en el caso mexicano (entre otras cosas por la inexistencia o poco acceso a archivos). Sin 
embargo, la subdisciplina ha contribuido significativamente a abrir la caja negra del Estado e incorporar actores y dinámicas nuevas para entender la política exterior. Así pues, no es extraño encontrar cada vez más textos que incorporan el papel de burocracias, grupos de interés, gobiernos subnacionales, osc, etc., en el diseño y la implementación de la política exterior. Aun así, la academia mexicana no ha logrado dar el salto entre un análisis conceptual de política exterior y una propuesta teórica de RR.II. o de Política Exterior alternativa a las existentes.

$$
x * *
$$

La disciplina de Relaciones Internacionales goza de buena salud en México, en el sentido de que es popular; se estudia y se enseña. Hay centros de estudio, departamentos y divisiones de estudios internacionales en un buen número de instituciones que ofrecen la licenciatura. Hay también publicaciones periódicas como Foro Internacional,Relaciones Internacionales, Política y Gobierno, Revista Mexicana de Política Exterior y Foreign Affairs Latinoaméri$c a$, entre otras, y universidades como el CIDE, el ITAM, El Colegio de México, la UNAM, y otras estatales publican el trabajo de sus investigadores. Además, los internacionalistas cuentan con la Asociación Mexicana de Estudios Internacionales (AMEI), fundada en 1967, que reúne no solo a académicos, sino también a miembros del servicio exterior y otros profesionistas interesados en asuntos internacionales. Según la información de su página electrónica, en la actualidad reúne a más de mil especialistas en RR.II. ${ }^{18} \mathrm{La}$ Asociación tiene una estrecha vinculación con la International Studies Association (ISA), con quien ha celebrado algunos de sus encuentros anuales; en ocasiones publica las memorias de sus congresos y otros textos de Relaciones Internacionales. Los congresos de la amei suelen ser el punto de encuentro de los internacionalistas no solo mexicanos, sino también extranjeros y, aún más importante, de los estudiantes de todo el país.

La salud, sin embargo, no es tan buena en referencia a la ausencia de contribuciones a la disciplina en general y a la ausencia de programas de posgrado, dos situaciones que están seguramente relacionadas. Como se ve en el Cuadro 1, hay pocas opciones para hacer maestrías o doctorados; los profesores-investigadores han sido educados en su mayoría en el exterior, en países del Norte, lo que sigue sucediendo con los egresados de la licenciatura. Es posible que este hecho contribuya a explicar por qué no hay producción teórica y metodológica propia. Es decir, limita la posibilidad de un pensamiento propio de RR.II. según la realidad de México y otros países periféricos, en especial América Latina. La pregunta obligada es por qué, dado el éxito de los programas de licenciatura, no hay más de posgrado.

18 https://www.amei.mx, consultada el 1 de octubre de 2019. 
No tengo una respuesta clara, pero imagino que es parte inercia y parte el dominio y prestigio de la academia sobre todo anglosajona. Es decir, caemos en un círculo vicioso. Igualmente, podríamos decir que la salud de la disciplina en México es un poco endeble en tanto sus programas siguen siendo muy tradicionales, como ya se ha mencionado.

Así pues, podría concluirse que el estudio de las Relaciones Internacionales en México ha sido una historia de éxito, vinculada estrechamente al desarrollo de la disciplina principalmente anglosajona pero que, para continuar así, enfrenta muchos retos: no solo continuar actualizándose ante un mundo extremadamente complejo, sino también, y más importante, proponerse seriamente tener una mirada propia. 